Mani pul at i on of aut or esonant intrinsi c I ocal i zed modes in MEMs arrays

\begin{tabular}{|l|l|}
\hline 著者 & $\begin{array}{l}\text { Sat o Nasayuki, Fuj i t a N., I mai S. , N shi mur a } \\
\text { S., Hor i Y., Si ever s A. J. }\end{array}$ \\
\hline $\begin{array}{l}\text { j our nal or } \\
\text { publ i cat i on ti tl e }\end{array}$ & Al P Conf er ence Pr oceedi ngs \\
\hline vol une & 1339 \\
\hline page r ange & $118-127$ \\
\hline year & $2011-$ O4 19 \\
\hline URL & ht t p: //hdl . handl e. net /2297/27838 \\
\hline
\end{tabular}




\title{
Manipulation of autoresonant intrinsic localized modes in MEMS arrays
}

\author{
M. Sato, N. Fujita, S. Imai, S. Nishimura, Y. Hori and A. J. Sievers
}

\begin{abstract}
The smallness of MEMS oscillators makes them an important platform for studying nonlinear phenomena. A unique feature, not shared by a harmonic system, is the dynamical steady state localization of some vibrations in driven nonlinear micromechanical lattices. Such an Intrinsic Localized Mode (ILM) is stable at any lattice site, and its position can be controlled by it interacting with an external field, a defect or another ILM. Both experiments and numerical simulations are used to explore the ILM-impurity attractive and repulsive interactions in micro-cantilever arrays in autoresonant states. The various findings reported here have a direct bearing on the application of nonlinear energy localization to implement smart functions in large-scale MEMS arrays.
\end{abstract}

\section{Introduction}

The small size of MEMS oscillators gives us a unique opportunity to study nonlinear dynamics because of the ease of exciting it into a nonlinear regime; for even if the total driving energy is small, the energy density will be large in a small system. Using a single oscillator platform fundamental nonlinear properties such as periodic attractors[1, 2] and stochastic resonances [3] have been investigated while at the same time applications such as sensors[4] and amplifiers[5] are being developed. Because of these possibilities, MEMS oscillators have been studied not only in single element form but also as coupled periodic arrays. Such micromechanical arrays provide a natural habitat for the study of dynamical localization of vibrations

M. Sato

Graduate School of Natural Science and Technology, Kanazawa University, Kanazawa, Japan email: msato@kenroku.kanazawa-u.ac.jp

A. J. Sievers

Laboratory of Atomic and Solid State Physics, Cornell University, Ithaca NY, USA e-mail: sievers@ccmr.cornell.edu 
in driven nonlinear lattices. An important property, not shared by a harmonic lattice, is the appearance of an Intrinsic Localized Mode (ILM)[6, 7, 8, 9, 10, 11, 12, 13]. In general a stationary ILM is stable in any lattice unit cell, but its position can be controlled by interacting with an external field, a vibrational defect mode or another ILM[7]. With the aid of an impurity mode different ILM processes have been identified, such as seeding, annihilating, repelling and attracting autoresonant ILMs.

In this paper first we experimentally explore controlling ILMs by introducing impurities into micro-cantilever arrays. The resulting attractive and repulsive impurityILM interactions that cause the ILM to move from one unit cell to the next are then analyzed in some detail using numerical simulations. Next by introducing a distribution of time dependent impurities in the lattice simulations are used to demonstrate ILM logic operations "NOR" and "OR". The end result of the different ILMimpurity perturbations is a variety of possible functions.

\section{Experimental observations of ILM position control by introducing an impurity}

For many of our experiments a di-element type array is used, consisting of a 300 $\mathrm{nm}$ thick cantilever elements on a silicon substrate. The elements are coupled one to another by a bridging silicon nitride overhang. The reason for a short and long cantilever in a unit cell is so the highest frequency plane wave mode can couple to the uniformly excited PZT attached to the silicon substrate. Since the sign of a cantilever onsite and intersite nonlinearities are both positive, the band frequencies increase as the displacement increases. Given the appropriate experimental conditions a nonlinear localized excitation, the ILM, will rises out of the top of the extended wave spectrum. Since energy damping naturally occurs continuous driving is required to maintain a steady state ILM. The end result is that the oscillation frequency is slightly higher than the top of the band, and this resulting ILM is continuously frequency locked to the driver[14]. In this autoresonant state[15] the amplitude of the ILM is controlled by the frequency of the driver. At the heart of the experimental measurement method is an optical lever. The tips of the cantilevers of the micromechanical array are illuminated by a line focused visible laser beam and the reflected light from each is recorded as a function of time using a one-dimensional CCD camera[16]. Figure 1 shows the experimentally observed ILMs for the micro-mechanical dielement cantilever array with hard nonlinearity. A stationary cantilever produces a signal at a pixel while a dark image identifies large amplitude motion of a cantilever tip so that the laser beam misses the camera. The image of cantilevers versus time is displayed in Fig. 1. The PZT is turned on at $t=0 \mathrm{~ms}$ and then its frequency is chirped up from the highest linear extended mode frequency (top of the band) to above it to reach the high amplitude autoresonant ILM state[15]. In the middle time interval of this figure there are many highly excited regions but after about $12 \mathrm{~ms}$ three stationary ILMs remain. As illustrated, the ILMs can appear at any short cantilever site. (The short cantilever sites are preferred because of the two cantilevers in 


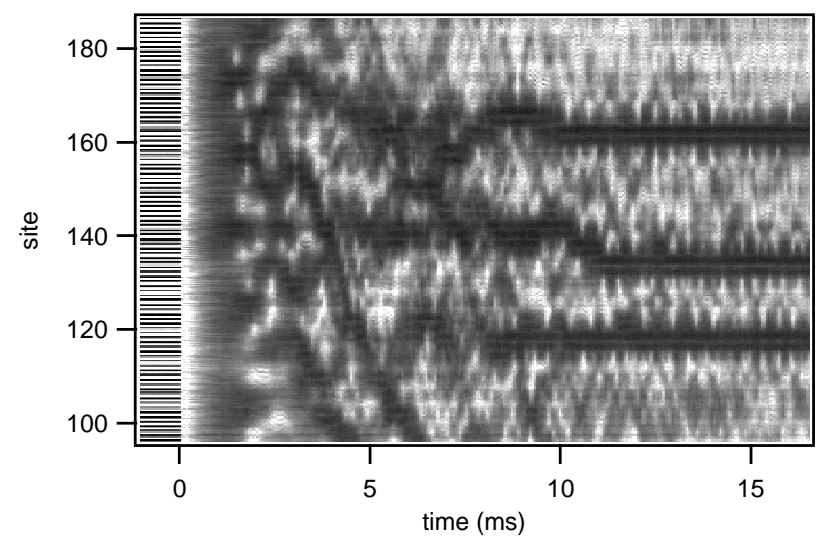

Fig. 1 Intrinsic localized modes in a cantilever array versus time. Top view of the cantilevers is shown on the left. Dark regions represent high amplitude vibrational states. These localized excitations are generated by chirping up the driver frequency above the highest band frequency of the linear spectrum. The driver is turned on at $t=0 \mathrm{~ms}$, and linear chirp end at about $1.6 \mathrm{~ms}$. The dark horizontal thick lines after about $12 \mathrm{~ms}$ represent stationary ILMs, where the amplitude is sufficient to pin the ILMs at a lattice site. In the intermediate time region they jumps between stable sites and collide with each other because of the relatively weak pinning effect associated with the smaller amplitudes.

a unit cell, it has the higher resonant frequency.) Although the ILM is translationally stable when its amplitude is large, in a collision with a movable excitation it may also move. The middle time region of the figure shows that for small ILM amplitude its mobility is significant. The next experiments involve the introduction and control of impurities in the lattice. The location of an ILM can be controlled by using an impurity mode outside of the extended wave frequency spectrum that is introduced externally[17]. Figure 2(a) shows the interaction of the ILM with an impurity produced by an IR laser beam. The strongly focused laser beam is introduced to heat up a few cantilevers and soften their harmonic restoring forces so that they form an impurity in the lattice. The resulting local mode has a frequency lower than the lowest extended mode of the branch. To record the experimental results shown in Fig. 2(a) first the focused laser beam is located nearby the ILM but at a small power level. Then, the power level of the IR laser is increased. The ILM is repelled and one picture is recorded. The figure is made by repeating the same sequence with the impurity at different spatial locations, demonstrating that an external probe can be used to move the ILM. Figure 2(b) demonstrates the capturing (tweezing) of an ILM using the same soft impurity produced by the IR laser as described above. The difference is in the array, which now supports soft (or negative) nonlinearity. This change in sign is produced by a thin electrode uniformly deposited over the cantilevers. When a DC voltage is applied between the cantilevers and the substrate the negative nonlinearity appears because of the resulting electrostatic attractive force between them. The impurity is then introduced as described above. 
Fig. 2 Top view of the ILM position versus frame number of the picture taken after each operation. Dark region (see arrow) identifies the ILM. Its position is controlled by a movable impurity. White rectangles are due to a focused IR laser beam that increases the temperature of a few cantilevers and lowers their resonance frequency resulting in a lattice impurity. (a) Moving the ILM with the repulsive interaction produced by a soft impurity in a hard nonlinear lattice. (b) Moving the ILM with the attractive interaction produced by a soft impurity in a soft nonlinear lattice.
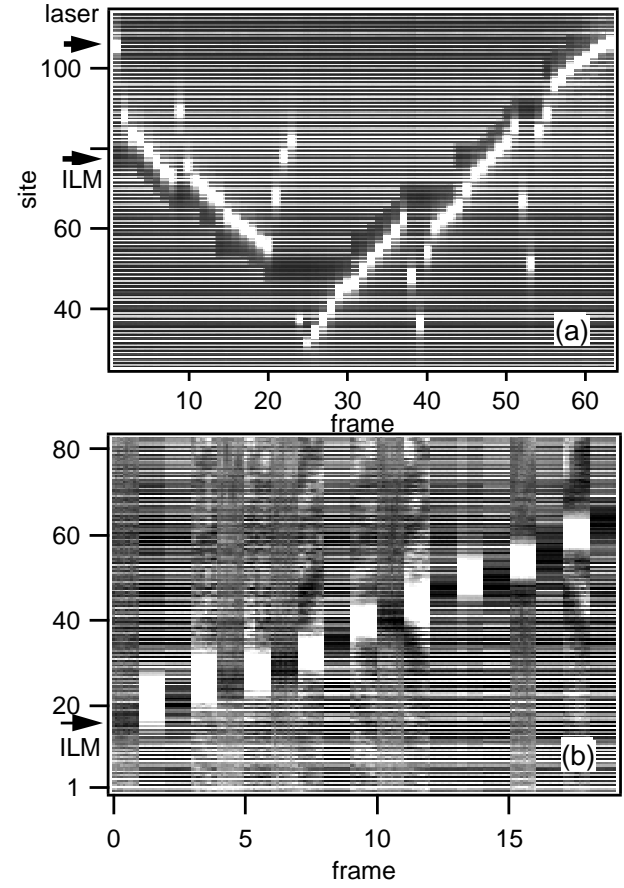

\section{Simulation of ILM position control with a lattice impurity}

To better understand the ILM-impurity interaction we use numerical simulations to explore the repulsive and attractive effects identified in Fig. 2. For these complimentary simulation studies we have found that a lumped ball and spring model provides a satisfactory representation of the nonlinear dynamics. Already treated is the case of a soft linear impurity, with the cantilever intrasite potential nonlinearity represented by either hard or soft quartic terms, respectively[7]. For the detailed simulations reported below the sign of both intrasite and inter site potential nonlinearities is always positive so the repulsive and attractive interactions are produced by either a soft or hard linear impurity.

The driven lumped element equation of motion for cantilever $i$ in a di-element array is of the form[7]

$$
\begin{aligned}
& m_{i} \ddot{x}_{i}+\frac{m_{i}}{\tau} \dot{x}_{i}+k_{2 O i} x_{i}+k_{4 O} x_{i}^{3} \\
& +\sum_{j} k_{2 I}^{(j)}\left(2 x_{i}-x_{i+j}-x_{i-j}\right) \\
& +k_{4 I}\left[\left(x_{i}-x_{i+1}\right)^{3}+\left(x_{i}-x_{i-1}\right)^{3}\right]=m_{i} \alpha \cos \omega_{d} t
\end{aligned}
$$


where $m_{i}$ is the mass, $\tau$ is a life time, $k_{2 O i}$ and $k_{4 O}$ are onsite harmonic and quartic spring constants, $k_{2 I}^{(j)}$ is a harmonic intersite spring constant of $j$-th nearest neighbor, $k_{4 I}$ is a quartic intersite spring constant, $\alpha$ is an acceleration, and $\omega_{d}$ is the driver frequency. The array is made from an alternative sequence of long and short cantilevers. Thus, $m_{i}$ and $k_{2 O i}$ are alternatively repeated along the array. The nonlinear components $k_{4 O}$ and $k_{4 I}$ are both positive and $k_{4 I}>>k_{4 O}$. The center of the ILM is at the short cantilever site (odd number site in the simulation). The driver frequency and amplitude are fixed $\omega_{d} / 2 \pi=139 \mathrm{kHz}$ and $\alpha=500 \mathrm{~m} / \mathrm{s}^{2}$. An impurity is introduced by changing $k_{2 O i}$ at a particular short cantilever lattice site.

Figure 3 shows the ILM changes that take place when a soft impurity $\left(k_{2 \mathrm{Oi}}^{\prime}<k_{2 \mathrm{O} i}\right.$ ) is slowly introduced into the lattice. The time dependence of the onsite force constant is given in Fig. 3(a). The initial lattice position of the ILM center at site 49, the position of the impurity (dotted line at site 47) and the final position of the ILM (site 51) are shown in Fig. 3(b). Note that with increasing time the amplitude of the ILM at site 50 grows while its amplitude at site 48 decreases. Since the ILM is in the autoresonant state its vibrational frequency is fixed in the run up to the transition to a new lattice site but the energy of this state is not fixed as is illustrated in Fig. 3(c). Since it requires more anharmonic energy at the impurity site than away from it

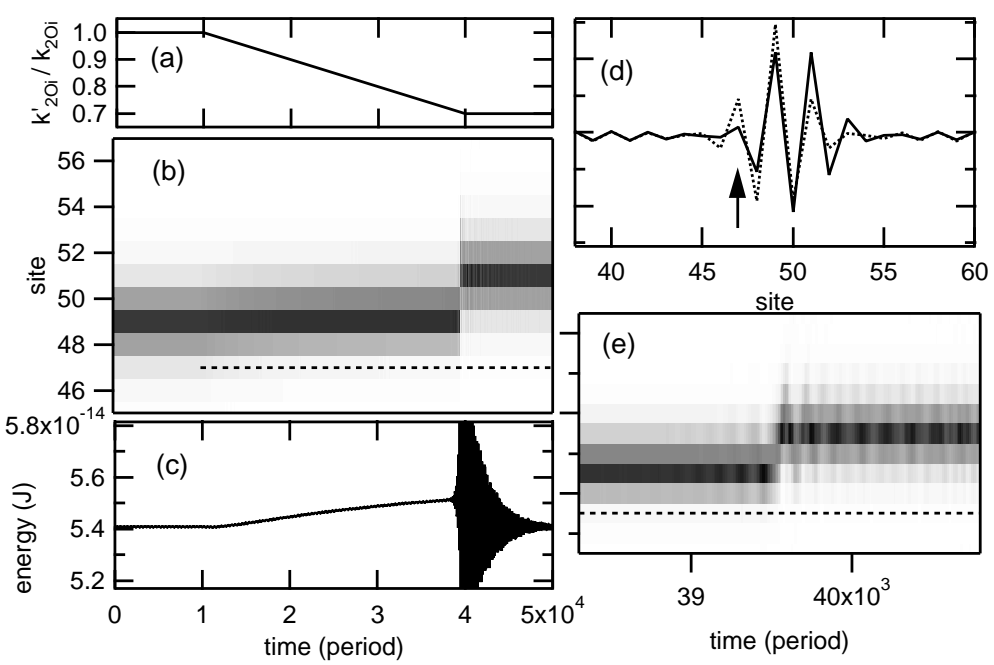

Fig. 3 Dynamics of the ILM during the repulsion by a lattice impurity. (a) Impurity time profile of an onsite spring constant $k_{2 O i}^{\prime} / k_{2 O i}$ at short cantilever site next of the ILM. (b) Initial ILM at site 49 . As the impurity is introduced at site 47 the ILM finally jumps to site 51. (c) Total energy of the ILM vs time. Energy gradually increases then returns to the initial value after the transition. Energy oscillations immediately after the transition are associated with lateral motion of the ILM. (d) Dotted trace; initial ILM eigenvector (single peaked); solid trace: ILM eigenvector (double peaked) just before the transition. The arrow identifies impurity position. (e) Magnified time scale near the transition. Oscillations in ILM amplitude both before and after the transition are associated with a lateral vibration induced by the transition. 
the ILM is repelled. The energy oscillations after the transition are associated with the energy going from the ILM into the lattice and back again to the ILM as it undergoes translational oscillations about the new equilibrium lattice site. Because of the small damping in the system it takes some time to reach the new steady state energy, which is the same as the initial ILM energy. Figure 3(d) presents eigenvector details of what unfolds locally. Before the impurity is introduced the vibrational eigenvector has the shape given by the dotted trace, where the center site has maximum amplitude. The solid trace shows the eigenvector after the impurity (see arrow) is introduced and just before the transition to the new lattice site. Now three sites have nearly the same amplitude. The initial energy increase is associated with the change in the eigenvector shape from single peaked to double peaked. This change in the eigen-form reduces the intersite potential barrier, facilitating the transition to the next lattice site. The final frame, Fig. 3(e), presents a snapshot of the transition over a smaller time scale. The lateral oscillations of the ILM around the new equilibrium position are evident. Note that a somewhat weaker version of these same lateral oscillations is evident before the transition. The excitation of this new degree of freedom is necessary; since, even in the transition region from single peaked to double peaked and back to single peaked again the ILM must remain in the same

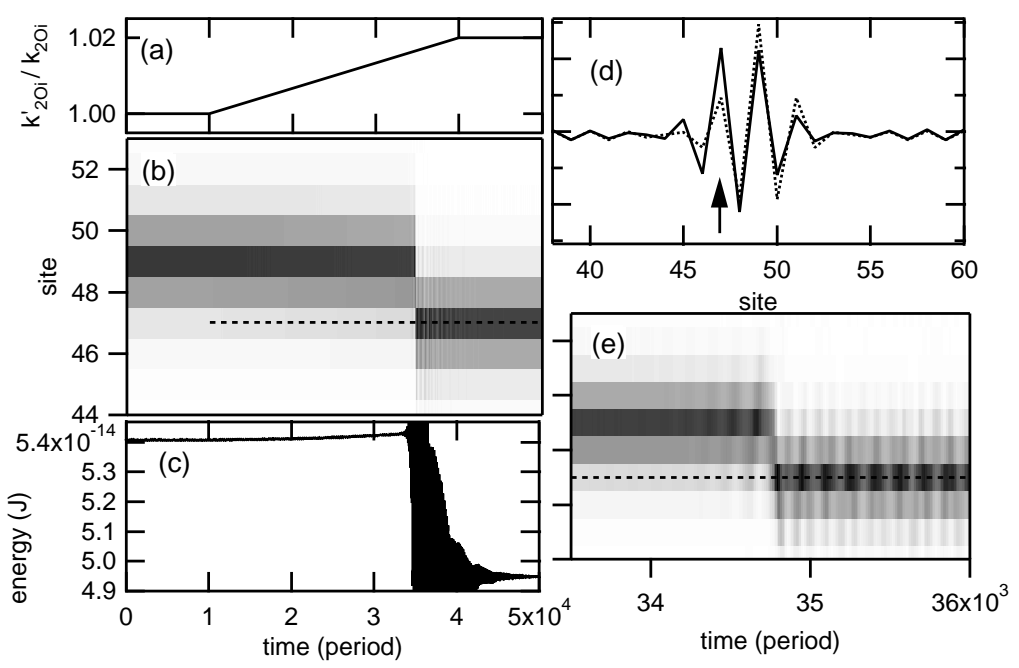

Fig. 4 Dynamics of the ILM during the attraction by a lattice impurity. (a) Impurity time profile of an onsite spring constant $k_{2 O i}^{\prime} / k_{2 O i}$ at short cantilever site next of the ILM. (b) Initial ILM at site 49. As the impurity is introduced at site 47 the ILM finally jumps to site 47 . (c) Total energy of the ILM vs time. Before the transition the energy increase is much smaller than for repulsive case. Very large lateral oscillations occur because of the large energy loss by the trapped ILM after the transition. (d) Dotted trace; initial ILM eigenvector (single peaked); solid trace: ILM eigenvector (double peaked) just before the transition. (e) Magnified time scale near the transition. Because of the large energy change during the transition lateral oscillations are excited involving more than one degree of freedom. 
fixed frequency autoresonant state. Because the ILM at the new site is farther from the impurity it has lower energy and the transition from the double peaked mode to the new single peaked mode is rapid.

The attractive interaction is more intriguing since it corresponds to capturing localized energy and moving it through the lattice with the aid of an impurity. Figure 4 shows the ILM changes that take place when a hard impurity $\left(k_{2 O i}^{\prime}>k_{2 O i}\right)$ is slowly introduced into the lattice. The onsite force constant now increases with time as illustrated in Fig. 4(a). The initial lattice position of the ILM center and the position of the impurity are the same as for the previous example. But now at the transition the ILM hops to the impurity site as shown in Fig. 4(b). In the run up to the transition Fig. 4(c) indicates that only a small increase in energy occurs as a function of time. The oscillations after the transition are associated with the energy going from the ILM into the lattice and back again to the ILM as it undergoes translational oscillations about the new equilibrium lattice site. Figure 4(d) presents the same initial single peaked eigenvector (dotted trace) as for the previous example, but now the eigenvector (solid trace) just before the transition, although again double peaked, has one maximum located on top of the impurity. Since it now requires less anharmonic energy at the impurity site than away from it the ILM is attracted to that site. The final frame, Fig. 4 (e), presents a snapshot of the transition over a smaller time scale. Note that there are now two different kinds of lateral oscillations of the ILM around the new equilibrium position. A somewhat weaker version of one of these same lateral oscillations also appears before the transition. Because of the large change in energy associated with the transition it is evident that two degrees of freedom have been excited in order to maintain the autoresonant state of the ILM during this large energy change.

The experiments described in Sect. 2 showed that for the case of hard ILM nonlinearity plus a soft harmonic impurity then repulsion occurred, while for the case of soft ILM nonlinearity plus soft impurity the result was attraction. In the simulations described above the case of hard nonlinearity plus a soft impurity was reproduced

Fig. 5 Impurity force constant profile and associated localized island modes. (a) Spatial distribution of $k_{2 O i}^{\prime} / k_{2 O i}$ profile. (b) Site dependence of island mode frequencies and amplitudes. Darker regions identify larger amplitudes. The modes below $137.1 \mathrm{kHz}$ are extended modes. 16 localized modes are induced by this distribution of impurities. The highest frequency impurity mode is close to the driver frequency $(139 \mathrm{kHz})$ for the simulation shown in Fig. 6.

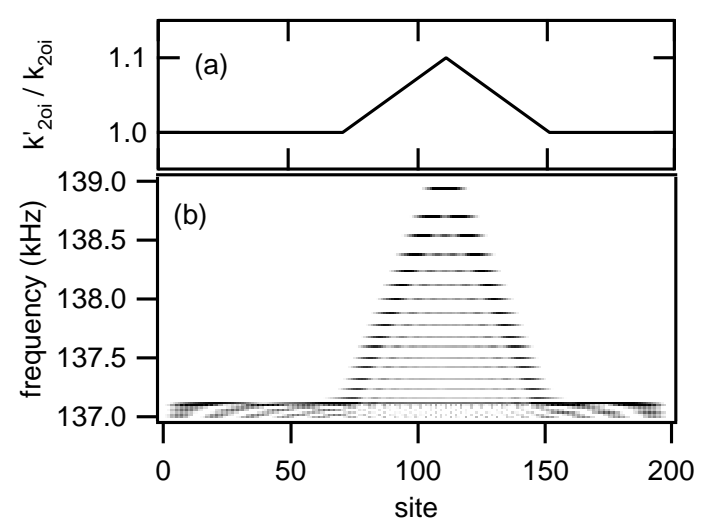


while the second simulation example was a case of hard nonlinearity plus hard impurity where attraction also was found. This second example was introduced here to illustrate that all of these cases demonstrate the same basic principle for the autoresonant state. If the anharmonic energy of the ILM is increased when the harmonic impurity approaches then the ILM is repelled while if the anharmonic energy of the ILM is decreased then attraction occurs.

\section{Simulation of an ILM-impurity island logic operation}

We have demonstrated how an impurity with a time dependent strength can be used to trap an ILM. A slow time dependence is required because the ILM is in an autoresonant state and any rapid variation would dislodge it from that condition. Rather than move the impurity to translate the attached ILM another method of locomotion is to introduce an impurity array. Here we introduce such a time and spatially dependent array to demonstrate how this same ILM-impurity interaction can be employed to produce a simple logic operation. The spatial dependence of the distributed set of harmonic spring constants for the impurities is shown in Fig. 5(a). It extends over a third of the lattice. At the time of its maximum application Fig. 5(b) displays the 16 highest frequency modes of the lattice. As illustrated these island modes are spatially localized. The remaining band of extended modes occurs below the lowest island mode.

We now illustrate two different ways to create an ILM in the perturbed lattice with hard nonlinearity. The driver frequency $(139 \mathrm{kHz})$ is set slightly above the top of the extended wave band $(137.1 \mathrm{kHz})$ and remains there through out the simulation. The driving amplitude is large enough to maintain an ILM once it is generated, but still small enough so that it cannot be generated directly without the assistance of the high frequency impurity mode. Method 1: With the impurities in place the highest frequency linear island mode resonates with the driver. By adiabatically lowering the strength of the impurities to zero the impurity resonance becomes an autoresonant ILM and remains at the same site. Method 2: Turn on the strength of the impurity distribution at a speed consistent with ILM relaxation time. Because the highest impurity mode frequency is tuned to the driver frequency, as shown in Fig. 5, resonance commences at the end of the ramp up. Now a localized pulsating structure appears at the center of the impurity distribution indicating amplitude modulation (AM) of the trapped excitation. Next remove the impurities in a time characteristic of the ILM relaxation time. Depending on the relative phase of the AM at the initiation of this removal process one finds either one ILM or no ILMs. The case for one ILM is shown in Fig. 6(a). The underlying dynamical behavior of the impurity modes makes this operation possible. The ILM is pulsing during the removal process, and the resulting phase difference changes the final ILM result.

In Figs. 6(b-c) the initial ILM is attracted toward the center of the impurity distribution. It collides with the seeded ILM at around $t=4000$ period and perturbs the pulsing phase of the ILM, changing it by 180 degrees. Similarly if the initial con- 


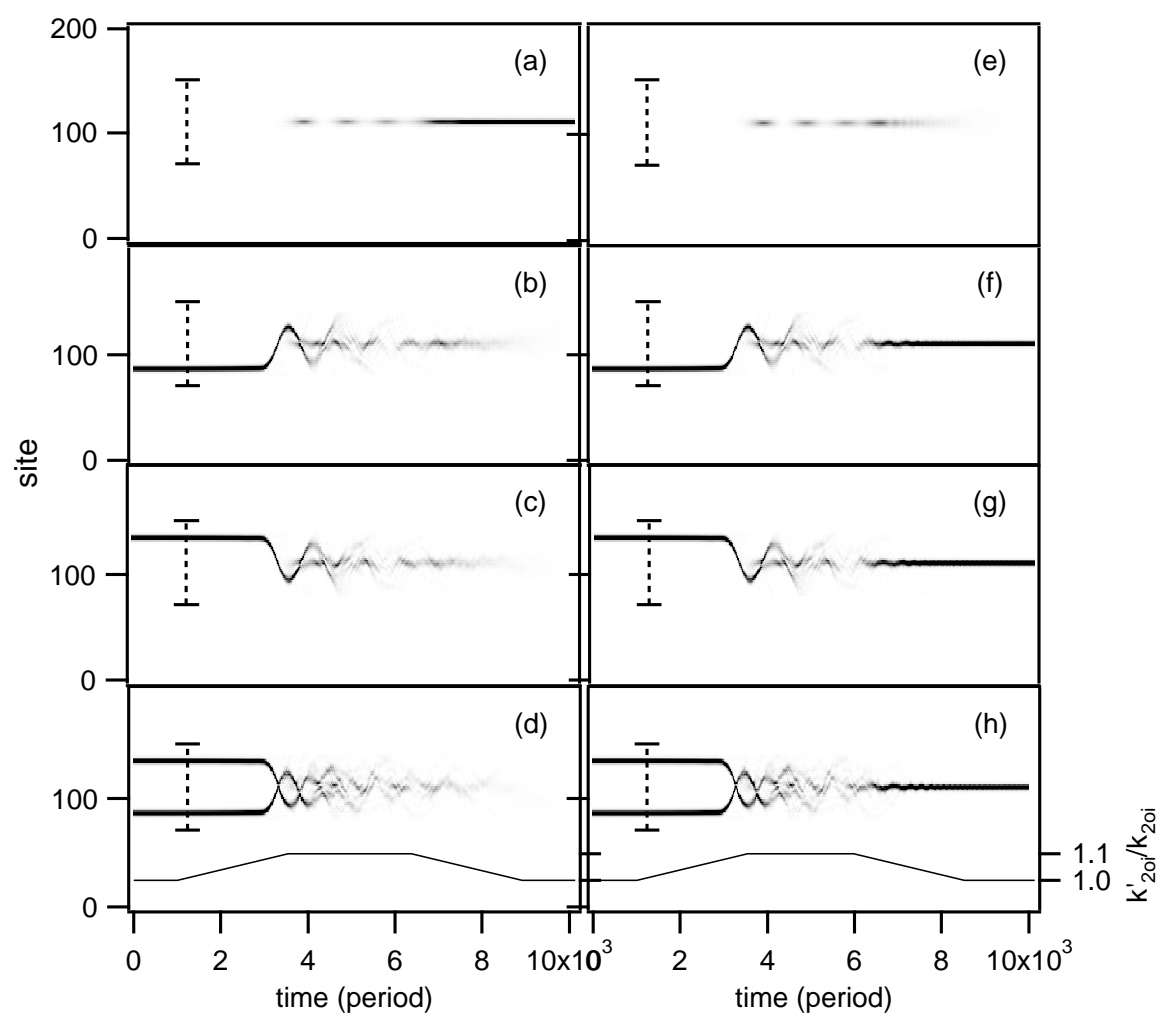

Fig. 6 Logic operation "NOR" demonstrated by simulations in a model for the hard nonlinear cantilever array. The existence and absence of the ILM correspond to logic "1" and "0", respectively. Input sites are 87 and 135 , output is site 111 . The same impurity operation is applied with different input conditions (a)-(d), where the number or location of input ILMs are different. Triangular distribution of impurity mode frequencies over sites 70-151 (dashed line) are applied as a function of time as shown by the solid curve in (d), where the spring constant of cantilever is modified as shown in Fig. 5. The ordinate for the impurity axis shows the ratio of the spring constant at the center impurity site. Among (a)-(d), no initial ILM cause the ILM output "1", that logic operation is "NOR". A pulsing oscillation of the ILM occurs during the operation. Its phase is modified and inverted by the existence of the initial ILM(s). The final result depends on the phase. In (e)-(h), the impurities are removed one half cycle earlier compared with cases in (a)-(d). Each output is the opposite from the corresponding case in (a)-(d). This operation is "OR".

dition is two ILMs placed equally on either side of the center of the impurity mode distribution as shown in Fig. 6(d) the pulsing phases of both ILMs are changed by 180 degrees. In all of the cases shown in Figs. 6(b)-(d) the end result is no ILM. Figure 6(e) through (h) show different time-profile cases. Here the removal of the impurities takes place one half cycle earlier than in Figs. 6(a)-(d). These frames show that the final state is the opposite from that shown in frames (a) through (d), in all cases the control variable is the phase of the pulsing ILM amplitude relative to 
the initiation time for removing the impurities. The end results depend on whether or not the ILM can continue to resonate with the driver as the extended impurity is removed.

In the series of simulations described here the speed of removing the impurities has been kept fixed, but the removal initiation time relative to the amplitude modulation signature is different. The opportunity to reach the high amplitude state (pure ILM) is favorable, if the impurity removal begins at an instant when the amplitude starts to increase. Conversely the no ILM state can be obtained by initiating impurity removal when the amplitude starts to decrease so that the impurity strength is reduced when the next AM maximum appears. If the modulation amplitude is too small, or if the impurity removal speed is too slow the end result is unchanged and the logic process is suppressed.

\section{Summary}

Our experiments with micro-cantilever arrays demonstrate both the production of ILMs and their control by space and time dependent impurities. The resulting attractive and repulsive ILM-impurity interactions are analyzed in some detail by using simulations. We have shown that the shape of the ILM eignevector is changed by the presence of a nearby impurity from a single-peaked to a double-peaked eigenmode signature, while the ILM itself continues to be locked to the driver frequency (autoresonance). As the eigenvector evolves, the energy increases until the transition to the new location takes place. If the anharmonic energy of the ILM increases when the harmonic impurity approaches then the ILM is repelled while if the anharmonic energy of the ILM decreases then attraction occurs.

By employing a time dependent distributed set of attractive impurities that induces many island impurity modes, the ILM logic operations "NOR" and "OR" have been obtained, The operation is based on switching into and out of the autoresonant state, and the pulsing behavior of the seeded ILM is produced by the rapid turn on of the impurity resonance. If the initiation time of the decreasing impurity strengths matches the phase of the increasing amplitude of the AM, the seeded ILM remains in auto-resonance, while it loses that state if the initiation time matches the phase of deceasing amplitude. It was also found that an existing ILM can modify the pulsing phase via the collision interaction between the initial ILM and the seeded one. It will be interesting to see if logic operations such as those mentioned here find applications in information processing, actuator arrays, and sensor arrays.

Acknowledgements This work was supported in part by JSPS Challenging Exploratory Research No.21656022 and NSF-DMR No. 0906491. 


\section{References}

1. J. S. Aldridge and A. N. Cleland, "Noise-Enabled Precision Measurements of a Duffing Nanomechanical Resonator," Phys. Rev. Lett. 94, 156403 (2005)

2. I. Kozinsky, H. W. C. Postma, O. Kogan, A. Husain, and M. L. Roukes,'Basins of Attraction of a Nonlinear Nanomechanical Resonator," Phys. Rev. Lett. 99, 207201 (2007)

3. M. I. Dykman, D. G. Luchinsky, R. Mannella, P. E. V. McClintock, N. D. Stein, and N. G. Stocks,"Supernarrow spectral peaks and high frequency stochastic resonance in systems with coexistings periodic attractors,"Phys. Rev. E 49, 1198-1215 (1994)

4. E. Buks and B. Yurke,"Mass detection with a nonlinear nanomechanical resonator," Phys. Rev. E 74, 046619 (2006)

5. I. Siddiqi, R. Vijay, F. Pierre, C. M. Wilson, L. Frunzio, M. Metcalfe, C. Rigetti, R. J. Schoelkopf, M. H. Devoret, D. Vion, and D. Esteve,'Direct Observation of Dynamical Bifurcation between Two Driven Oscillation States of a Josephson Junction," Phys. Rev. Lett. 94, 027005 (2005)

6. M. Sato, B. E. Hubbard, A. J. Sievers, B. Ilic, D. A. Czaplewski, and H. G. Craighead,"Observation of locked intrinsic localized vibrational modes in a micromechanical oscillator array," Phys. Rev. Lett. 90, 044102 (2003)

7. M. Sato, B. E. Hubbard, and A. J. Sievers,"Nonlinear energy localization and its manipulation in micromechanical oscillator arrays," Rev. Mod. Phys. 78, 137-157 (2006)

8. P. Maniadis and S. Flach,"Mechanism of discrete breather excitation in driven micromechanical cantilever array," Europhys. Lett. 74, 452-458 (2006).

9. A. J. Dick, A. J. Balachandran, and C. D. Mote,'Intrinsic localized modes in microresonator arrays and their relationship to nonlinear vibration modes," Nonlin. Dyn. 54, 13-29 (2008)

10. Q. Chen, L. Huang, Y.-C. Lai, and D. Dietz,'Dynamical mechanism of intrinsic localized modes in micromechanical oscillator arrays," Chaos 19, 013127 (2009)

11. E. Kenig, R. Lifshitz, and M. C. Cross,'Pattern selection in parametrically driven arrays of nonlinear resonators " Phys. Rev. E 79, 026203 (2009)

12. E. Kenig, B. A. Malomed, M. C. Cross, and R. Lifshitz, "Intrinsic localized modes in parametrically-driven arrays of nonlinear resonators," Phys. Rev. E 80, 046202 (2009)

13. J. Wiersig, S. Flach, and K. H. Ahn,'Discrete breathers in ac-driven nanoelectromechanical shuttle arrays" Appl. Phys. Lett. 93, 222110 (2009)

14. T. Rössler and J. B. Page,'Intrinsic localized modes in driven anharmonic lattices with realistic potentials," Phys. Lett. A204, 418-426 (1995)

15. J. Fajans and L. Friedland,"Autoresonant (nonstationary) excitation of pendulums, Plutinos, plasmas and other nonlinear oscillators," Am. J. Phys. 69, 1096-1102 (2001)

16. M. Sato and A. J. Sievers," Visualizing intrinsic localized modes with a nonlinear micromechanical array," Low Temperature Physics 34, 543-548 (2008)

17. M. Sato, B. E. Hubbard, A. J. Sievers, B. Ilic, and H. G. Craighead,"Optical manipulation of intrinsic localized vibrational energy in cantilever arrays," Europhys. Lett. 66, 318-323 (2004) 